\title{
An Instrument for Measuring Longitudinal Spherical Aberration of Lenses
}

\author{
By Francis E. Washer
}

\begin{abstract}
An instrument is described that permits the rapid determination of longitudinal spherical and longitudinal chromatic aberration of lenses. Specially constructed diaphragms isolate successive zones of a lens, and a movable reticle connected to a sensitive dial gage enables the operator to locate the successive focal planes for the different zones. Results of measurement are presented for several types of lenses. The instrument can also be used without modification to measure the small refractive powers of goggle lenses.
\end{abstract}

\section{Introduction}

In the course of a research project sponsored by the Army Air Forces, an instrument was developed to facilitate the inspection of lenses used in the construction of reflector sights. This instrument enabled the inspection method to be based upon a rapid determination of the axial longitudinal spherical aberration of the lens. This measurement was performed with the aid of a series of diaphragm plates that enabled the operator to select any zone of the lens at will and quickly determine the separation of its focal plane from that of any other zone by movement of a reticle whose movements were registered by a sensitive dial gage.

The instrument proved so simple and accurate that a second one has been constructed for use in measuring axial longitudinal spherical and longitudinal chromatic aberration of lenses. In addition, the instrument can be readily used in the measurement of small refractive powers in the region from -0.06 to +0.06 diopters. It is therefore extremely useful, in the determination of the refractive power of goggle lenses in the course of tests, to determine compliance with federal specifications.

\section{Apparatus}

The apparatus consists of a movable, illuminated reticle, a sensitive gage to record the movement of the reticle, a collimating lens, one movable and one fixed diaphragm, and a viewing device.
The arrangement of these elements is shown diagrammatically in figure 1 . To simplify the description, the optical system will be considered in three parts, each of which forms a definite member of the whole system. The axis, $A$, is common to the entire system; the axis is bent at the mirror, $M$, to conserve space and simplify operation by a single observer.

Objective lens $L_{1}$, mirror $M$, and viewing screen, $S$ form the first part. The viewing screen, $S$, lies in the focal plane of lens $L_{1}$, so that light, parallel to the axis, $A$, incident on the front of $L_{1}$, passes through it, is reflected by $M$, and is brought to a focus at $S$. The screen, $S$, may be replaced by an ocular, so that the combination of $L_{1}, M$, and the ocular form a telescopic system focused for parallel light. Mirror $M$ is a plane frontsurface mirror mounted in a fixture equipped with adjusting screws, and its function is to bring the image formed by $L_{1}$ to the center of screen $S$. The choice of the focal length of lens $L_{1}$ is determined by several factors. These are easy viewing, ready observation of small lateral shifts of the image, and the miniíying of error in $L_{1}$. A lens having a focal length of $1,143 \mathrm{~mm}$ is used at present in the instrument. Accordingly, an angular change of $1 \mathrm{mil}$ in the light incident upon $L_{1}$ produces a lateral displacement of approximately $1.14 \mathrm{~mm}$ of the image formed by $L_{1}$ on screen $S$. The long focal length of $L_{1}$ insures negligible error of parallax from any residual longitudinal spherical aberration in $L_{1}$. 


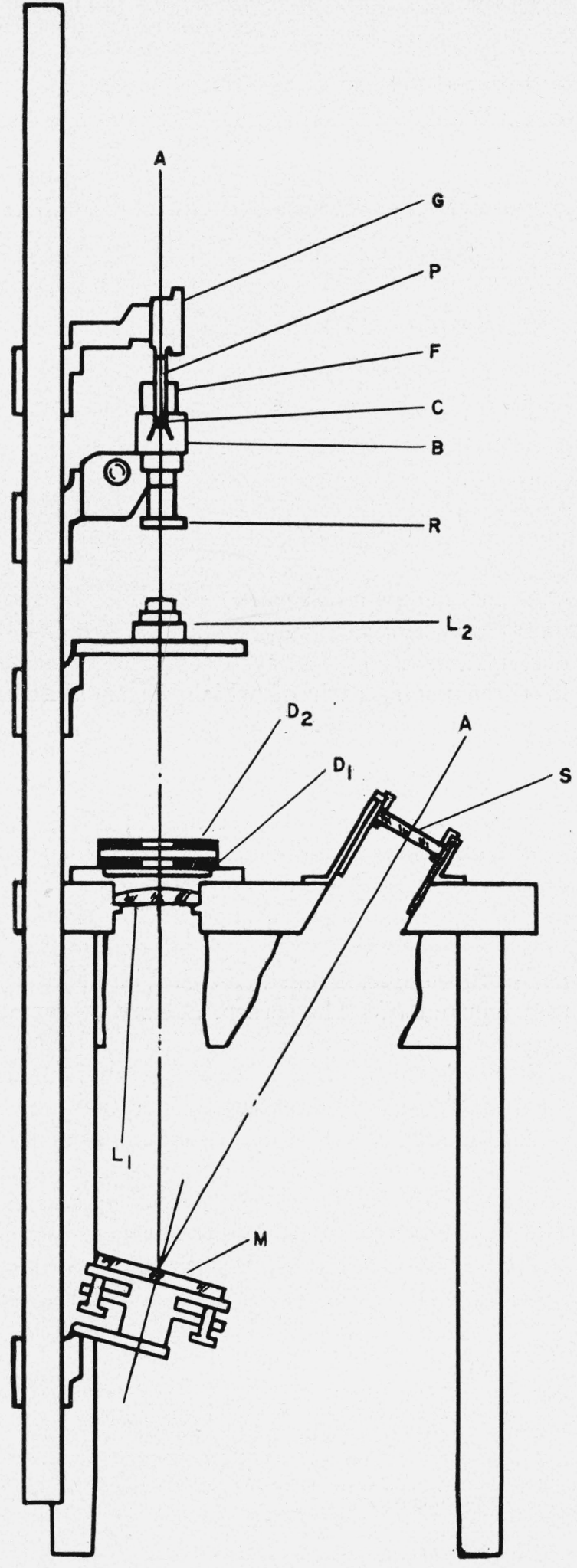

Figure 1. Diagrammatic sketch of the instrument.
Lens $L_{2}$, which frequently is a lens corrected for parallel incident light, and the diaphragms, $D_{1}$ and $D_{2}$, form the second part of the system. $L_{2}$ is the lens to be tested and is mounted over an opening in the supporting plate. The supporting plate is provided with adjustable brackets that facilitate easy insertion, proper positioning, and easy removal of the lens. Lens $L_{2}$ is so placed that its "infinite conjugate" is facing lens $L_{1}$. Diaphragm $D_{1}$ contains a rectangular slot whose length is equal to the effective aperture of $L_{1}$ and

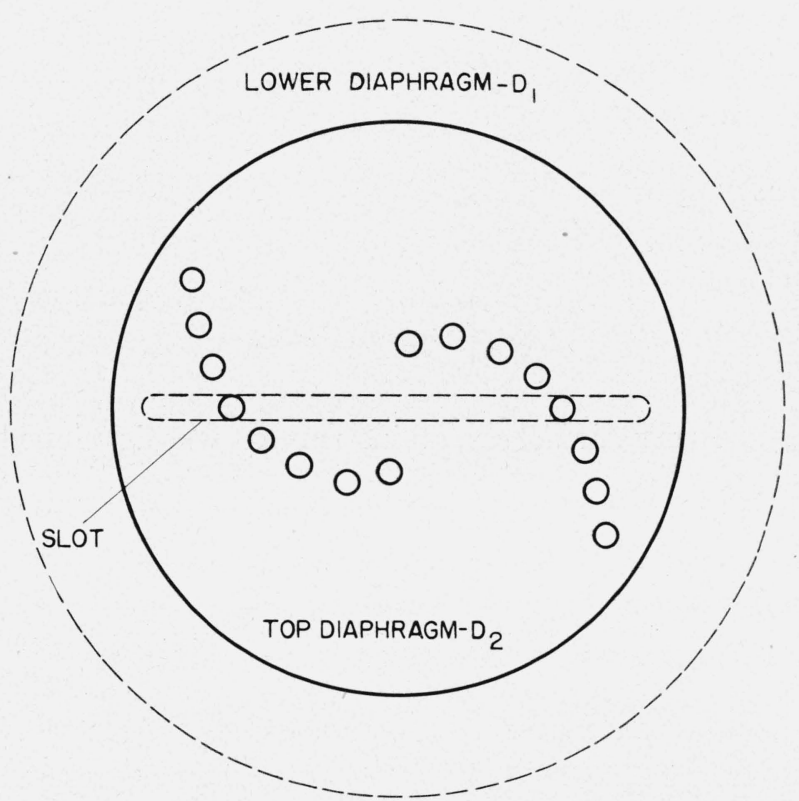

FIGURE 2. Diagrammatic sketch showing the manner in which the successive pairs of holes in the upper diaphragm, $D_{2}$, are isolated by the slot in the lower diaphragm, $D_{1}$.

whose width is approximately $5 \mathrm{~mm}$. Its function is to isolate a narrow region along the diameter of $L_{2}$. Diaphragm $D_{2}$ is located above $D_{1}$ and contains circular holes of small diameter arranged in pairs about the center of the diaphragm. The arrangement of these pairs of holes is shown in figure 2. The two members of a pair are equidistant from the center and on the same diameter. The linear separation of the members of a pair proceeds in steps of $5 \mathrm{~mm}$ from 5 to $100 \mathrm{~mm}$. Rotation of the diaphragm $D_{2}$ above $D_{1}$ therefore brings successive pairs of holes over the slot in $D_{1}$ and so isolates successive zones of lens $L_{2}$. In order to provide a sufficient number of holes, so spaced that only one pair is above the opening in $D_{1}$ at a time, it is necessary to have several interchangeable diaphragms that can be used at 
$D_{2}$. Each of the diaphragm plates has from two to eight pairs of holes.

The reticle, $R$, lamp $F$, movable member $B$, and dial gage $G$ form the third part of the system. Member $B$ is movable in a vertical direction by means of a rack and pinion. The friction opposing the movement is sufficiently great to prevent appreciable drift between adjustments. Member $B$ supports a lamp house containing lamp $F$ and reticle $R$. Reticle $R$ is a transparent cross and is mounted on a support that permits small displacements necessary for centering. Reticle $R$ is illuminated by lamp $F$ and is so situated that its center lies at or near the focal point of lens $L_{2}$. The upper portion of $B$ is provided with a cap that transmits its vertical motion to the sensitive length-measuring gage, $G$, through plunger $P$. Thus, any raising or lowering of reticle $R$ is done by moving $B$, and the magniture of the movement is indicated on gage $G$.

Figure 3 is a photograph of the actual instrument showing the manner in which the apparatus is arranged for easy use by a seated observer. The ocular assembly is removable and can be replaced by a ground-glass viewing screen. Telescope lens, $L_{1}$, mounted in the opening in the table top, can be focused by rotating the ocular mount or by sliding the mirror mount, $M$, up or down the supporting shaft. The other elements of the system are attached to the upright shaft by clamps that can be moved up and down by large amounts and so permit the use of a wide variety of collimating lenses with focal lengths ranging from 1 to $30 \mathrm{in}$. The lamp house includes a prism, movable condensers, and filter holders to provide controlled illumination of the reticle. The collimating lens support is provided with a series of attachments, so that lenses having diameters ranging from 10 to $100 \mathrm{~mm}$ may be firmly held.

\section{Theory of the Instrument}

Longitudinal spherical aberration is the variation in focal length with differing annular zones of a lens. To measure the spherical aberration, it is customary to intercept parallel light before incidence on the lens by an appropriate diaphragm that limits the transmitting area of the lens to a selected annular zone; the position of the focal plane for this particular zone is then determined.

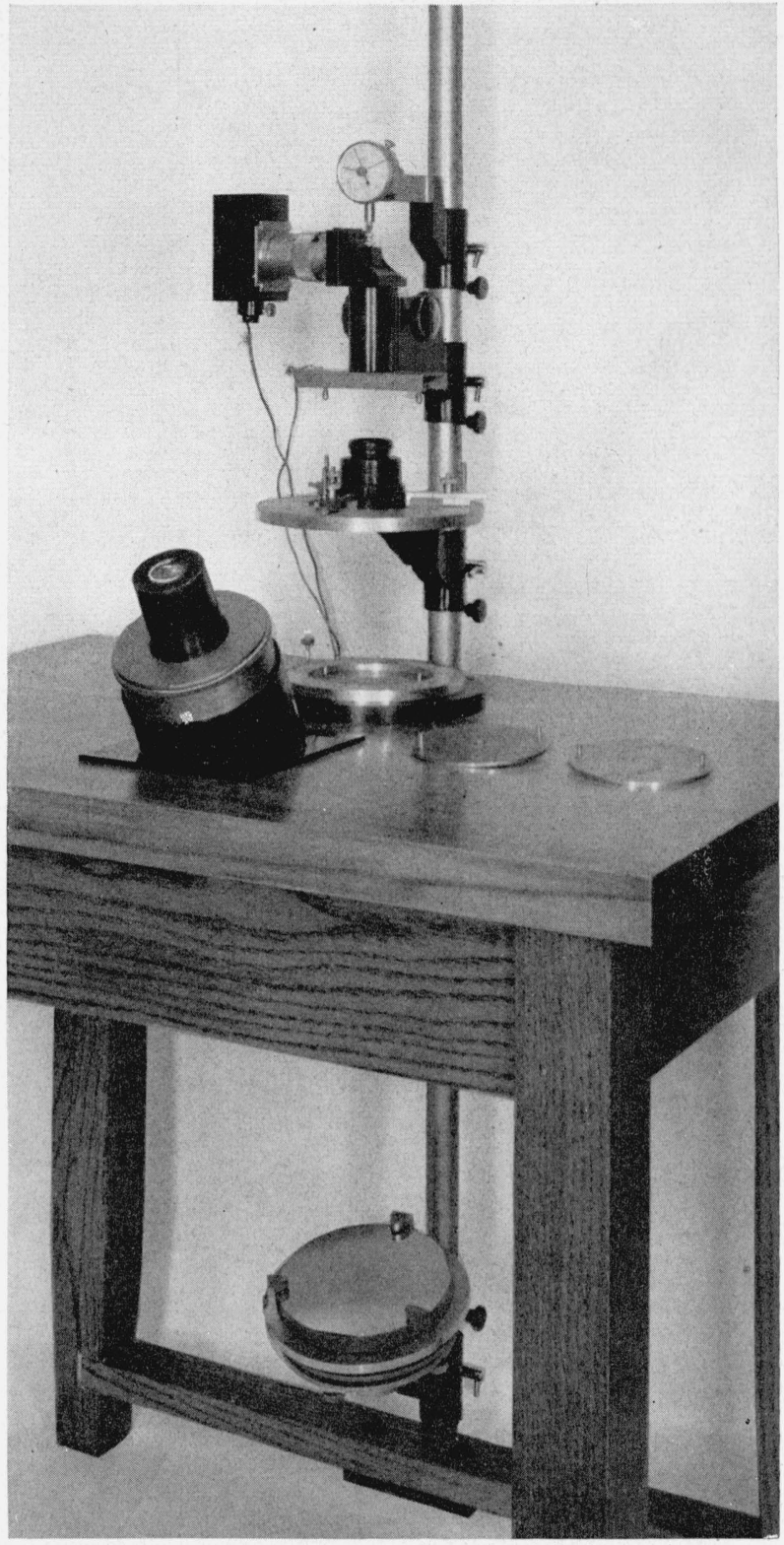

FiguRe 3. Photograph showing the instrument and two of the auxiliary diaphragms.

The process is repeated for a series of diaphragms that successively isolate each annular zone until the entire surface has been covered. The position of the focal plane is determined for each of these zonal diaphragms with respect to the position determined for the first diaphragm. The resulting values of change in focus $\Delta f$ are plotted against zone radius $r$ and the typical longitudinal spherical-aberration-curve results.

The changes in focal length for successive zones of the lens under test in the foregoing method are 
measured on a lens bench, using a moving microscope to observe the successive positions of best focus for the various zones. A collimator serves as the source of parallel light.

In the present instrument the light path is reversed. The collimator target is replaced by a viewing screen at the plane of focus for parallel light, or by an ocular so placed that the combination of viewing lens and ocular form a telescopic system. The moving microscope is replaced by a movable reticle whose displacements are registered on a sensitive dial gage. Light from the illuminated reticle passes through the lens under test and is properly imaged on the viewing screen only when the reticle is in the foeal plane of the lens under test. When this condition is presented, parallel light is emerging from the lens under test. The isolation of successive zones of the lens under test is accomplished by the paired holes in diaphragm $D_{2}$.

In figure 4, a schematic drawing of the light paths through the instrument is shown. Light

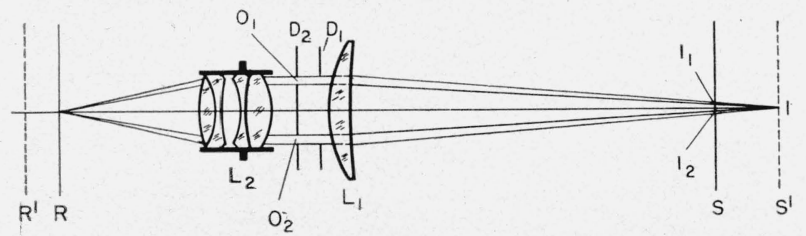

FIGURE 4. Light paths through the instrument when reticle $R$ is nearer to the collimating lens, $L_{2}$, than the focus of the zone determined by the diaphragm openings, $\mathrm{O}_{1}$ and $\mathrm{O}_{2}$. $R$ must move to $R^{\prime}$ in order that a single image he seen on screen $S$.

from the reticle $R$, which is near the true focal plane of the lens, passes through lens $L_{2}$. The diaphragms, $D_{2}$ and $D_{1}$, intercept all light emerging from $L_{2}$, except for two small cylindrical beams that pass through the small openings, $O_{1}$ and $O_{2}$. These beams would normally be brought to a focus by lens $L_{1}$ at $I$ in the plane, $S^{\prime}$. However, the viewing screen, $S$, lying in the focal plane of $L_{1}$, intercepts the light so that the observer sees two images, $I_{1}$, and $I_{2}$, on screen $S$. Definite images are formed at $S$ even though $S$ is appreciaably distant from the true image plane, $S^{\prime}$. This is possible, as the beams of light passing through $O_{1}$ and $O_{2}$ are finite in size, and each beam is capable of forming an image of reticle $R$. The depth of focus is so great at small apertures that distinct images, $I_{1}$ and $I_{2}$, are seen at $S$, even though $S$ does not coincide with the plane of best imagery.
Movement of reticle $R$ along the axis of lens $L_{2}$ causes the images, $I_{1}$ and $I_{2}$, to separate from or to aproach each other, depending upon the direction of movement of $R$. In the present instance, if $R$ moves to $R^{\prime}$, the two images, $I_{1}$ and $I_{2}$, coalesce to form a single image at $S$. If $R$ moves beyond $\mathrm{R}^{\prime}$, the images will again separate, but $I_{1}$ and $I_{2}$ will interchange their positions and $I$ would lie between $L_{1}$ and $S$. When $R$ and $R^{\prime}$ coincide, the reticle is at the focus of lens $L_{2}$ for the given zone, the beams of light passing through the openings $O_{1}$ and $O_{2}$ are parallel to each other and to the optic axis, and a single image appears on screen $S$. The reading on gage $G$ is recorded, and a second pair of holes in $D_{2}$ is brought over the slot in $D_{1}$. The process of locating a position of $R$ such that a single image appears at $S$ is repeated, and the reading of gage $G$ is recorded. The difference between the successive readings of the gage is the longitudinal spherical aberration, $\Delta f$, that exists between the two selected zones of the lens. This procedure can be extended for a sufficient number of zones to cover the entire lens and the complete longitudinal spherical-aberration data obtained.

\section{Results of Measurement}

The longitudinal spherical and longitudinal chromatic aberration of a group of lenses having focal lengths varying from 3 to 13.5 inches have been measured with this instrument. The lenses are of various types and include telescope objectives, photographic objectives, and collimating or projection lenses. The results of measurements are shown graphically in figures 5 to 9 . The zone radius is plotted as ordinate against the variation in focal length as abscissa.

In making these measurements, a set of diaphragm plates are used that permit isolation of successive zones at $2.5-\mathrm{mm}$ intervals of zone radius, from the smallest for which fission of the image occurs to the maximum radius of the lens aperture. Wratten monochromatic filters Nos. $71 \mathrm{~A}$ (Orange Red), 73 (Yellow Green), and 75 (Blue Green) provide a sufficient range of color separation to illustrate the nature of the longitudinal chromatic aberration.

In plotting the curves in figures 5 to 9 , the values of $\Delta f$, corresponding to a given zone radius, are shown as circles for filter No. 75, as crosses for filter No. 73, and as solid circles for filter No. 71A. 


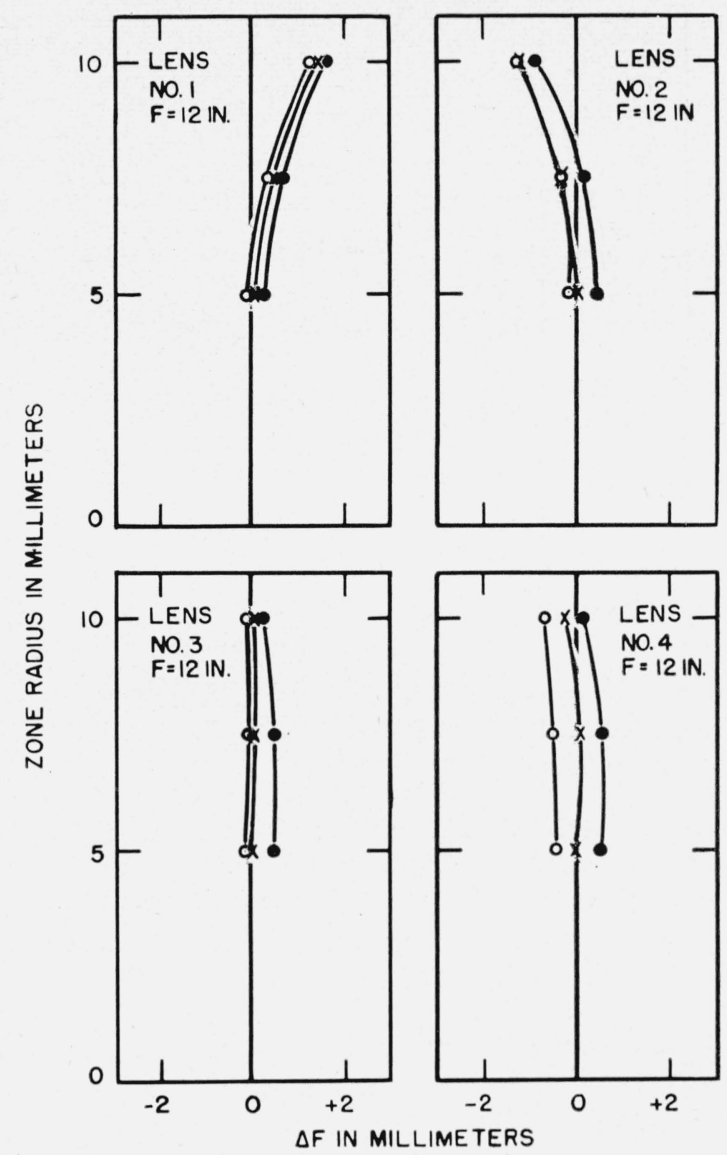

Figure 5. Axial longitudinal spherical- and chromaticaberration curves for a group of four telescope objectives.

The open circles are determinations made with Wratten filter No. 75 , the crosses with Wratten filter No. 73, and the solid circles with Wratten filter No. 71A. Lens No. 3 is a well-corrected lens having a flint front element, lens No. 1 is over-corrected spherically, lens No. 2 is not corrected for spherical aberration, and lens No. 4 is not correeted for axial chromatic aberration.

If a lens had no longitudinal spherical aberration, the resulting curve for a given filter would be a straight line parallel to the axis of ordinates. If a lens had zero longitudinal chromatic aberration, the points for the various colors of light for a given zone radius would coincide, and there would be no separation of the curves. However, these aberrations are always present to some extent in actual lenses, so that the maximum separation of points on a given curve measured along the axis of abscissae may be used to judge the excellence of the longitudinal spherical correction. The separation of the curves for the different colors is a measure of the amount of longitudinal chromatic aberration present in the lens.

The results shown in figure 5 are for a group of telescope lenses belonging to a laboratory demon- stration set. Lens No. 1 is over-corrected spherically, which is evidenced by the curve, the focal length for the outer zone being approximately $1.5 \mathrm{~mm}$ larger than that for the innermost zone shown. Lens No. 2 is not spherically corrected and in consequence has an appreciably shorter focal length for the outer zone. Lens No. 3 is a wellcorrected lens having a flint front element; it is clear from the flatness of the curves that the longitudinal spherical aberration is negligibly small, although there is appreciable longitudinal chromatic aberration as is shown by the separation of the curves. Lens No. 4 is not chromatically corrected, and all three of the longitudinal spherical aberration curves are well separated.

Figures 6,7 , and 8 illustrate the results obtained on photographic objectives of various focal lengths and types. Lens No. 6 in figure 6 is a telephoto lens having a large amount of spherical aberration. Lens No. 5 is a photographic objective having a moderate amount of spherical aberration. Lenses

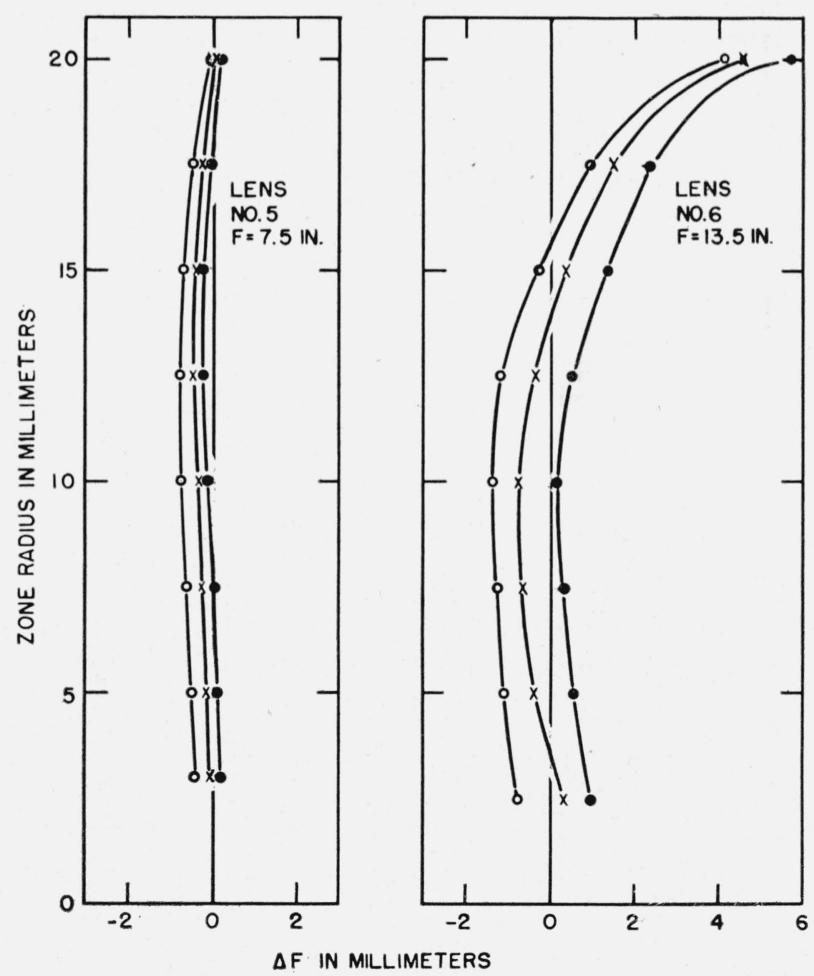

FIgUre 6. Axial longitudinal sphericai- and chromaticaberration curves for two photographic objectives.

The open eircles are determinations made with Wratten filter No. 75 , the crosses with Wratten filter No. 73, and the solid circles with Wratten filter No. 71A. Lens No. 6 is a telephoto lens having a large amount of spherical aberration, and lens No. 5 is a photographic objective having a moderate amount of spherical aberration. 
Nos. 7 and 8 in figure 7 are of the same type but of different focal lengths; the amount of spherical aberration is great enough to produce noticeable change in focus when this type of lens is stopped down. Lenses Nos. 9 and 10 in figure 8 have so little spherical aberration that it is necessary to magnify the scale of abscissae to show it. However, the testing instrument is sufficiently sensitive that even these small amounts are readily measurable.

The spherical aberration of lens No. 11 is shown in the left-hand portion of figure 9. Lens No. 11 is a short-focus collimating lens and has a considerable amount of axial astigmatism. This is shown in the right half of figure 9 . The measurements for curve 2 are taken along a diameter of the lens at right angles to the diameter used for curve 1. There are marked differences in the focal length through the range of zone radii. These two curves illustrate the capacity of the instru-

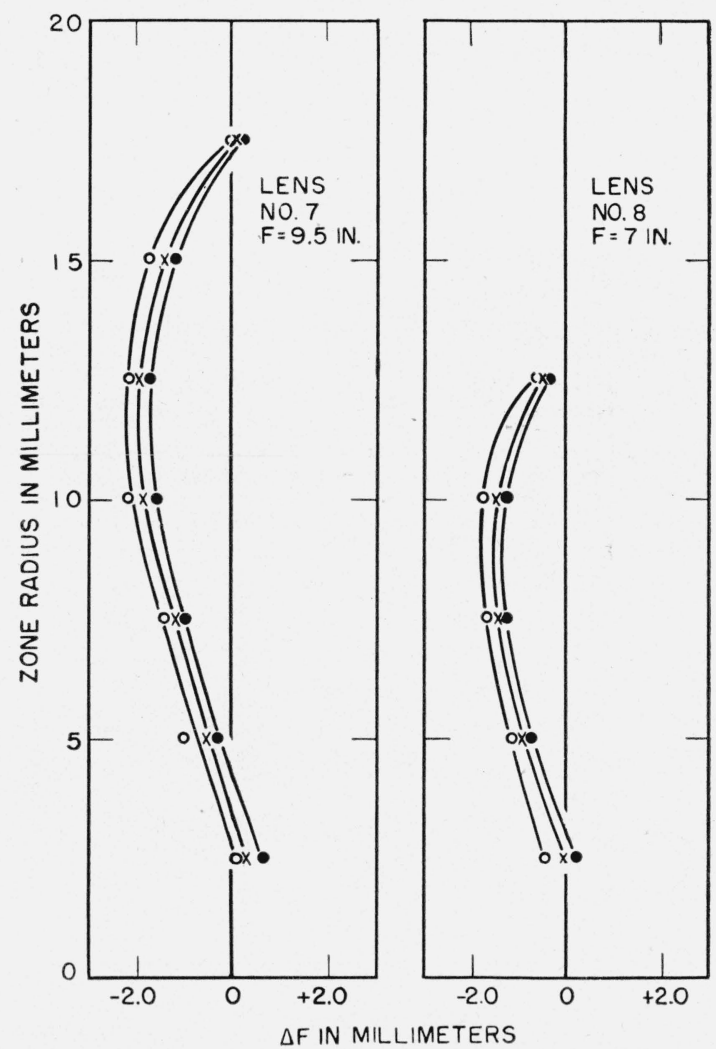

Figure 7. Axial longitudinal spherical and axial chromatic aberration curves for two photographic objectives of the same type but having different focal lengths.

The open circles are determinations made with Wratten filter No. 75, the crosses with Wratten filter No. 73, and the solid circles with Wratten filter No. $71 \mathrm{~A}$.

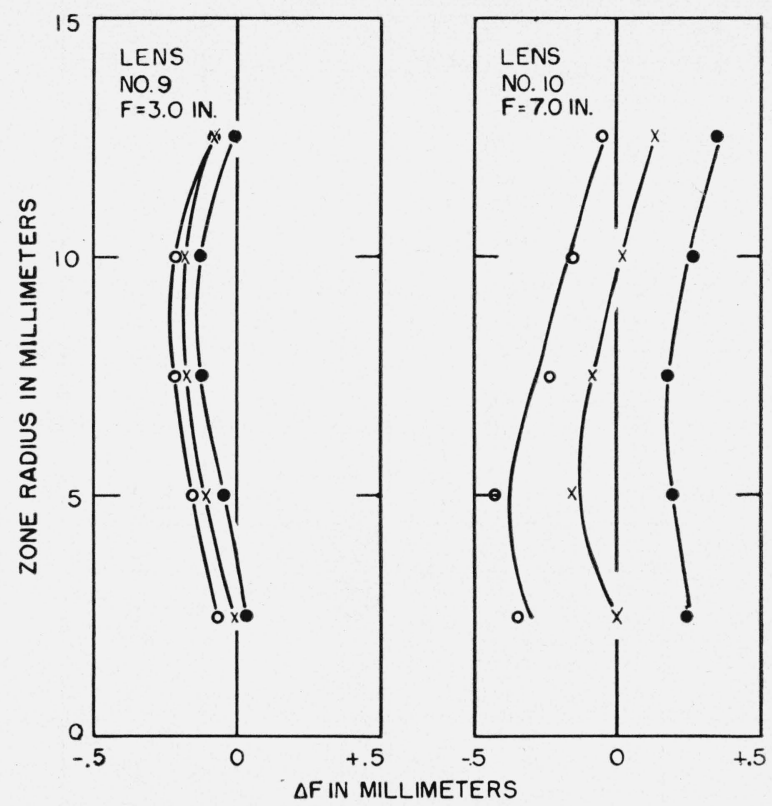

Figure 8. Axial longitudinal spherical and axial chromatic aberration curves for two photographic objectives that have very smail amounts of spherical aberration.

The open circles are determinations made with Wratten filter No. 75 , the crosses with Wratten filter No. 73 , and the solid circles with Wratten filter No. $71 \mathrm{~A}$.

ment to detect and measure astigmatic effects in lenses.

The accuracy with which the values of $\Delta f$ can be measured with this instrument depends upon the $f$-number of the lens for the selected zone and the quality of the imagery. For good-quality imagery the probable error in millimeters of a single observation seldom exceeds \pm 0.004 times the $f$-number. Accordingly, the accuracy varies from $\pm 0.02 \mathrm{~mm}$ for a zone with aperture ratio $\mathrm{f} / 5$ to $\pm 0.10 \mathrm{~mm}$ for a zone with aperture ratio $\mathrm{f} / 25$. The values of $\Delta f$ can therefore be measured with good precision over most of the effective area of the lens, and as the depth of focus is so great at the large $f$-numbers, the accuracy of the method is adequate for most purposes even at the large $f$-numbers.

\section{Additional Uses of the Instrument}

The present instrument is the second to be constructed. The first instrument was built for the Army Air Forces and was primarily intended for use in measuring the parallax arising from longitudinal spherical aberration present in collimating lenses of the type used in reflector sights. During the course of investigation of the properties 
of the primary instrument, it was found that the instrument was ideally suited for the rapid measurement of small refractive powers of goggle lenses in addition to its principal task of measuring longitudinal spherical aberration in connection with the computation of parallax. This section therefore discusses the manner in which the instrument is used to measure small refractive powers and the manner in which it is used to measure parallax of collimating lenses.

\section{Measurement of Small Refracting Powers}

One of the constantly recurring tasks at this Bureau is the measurement of the spherical refracting power of eye-protective lenses having, nominally, zero refractive power to determine compliance with the optical requirements contained in federal specifications. The tolerances

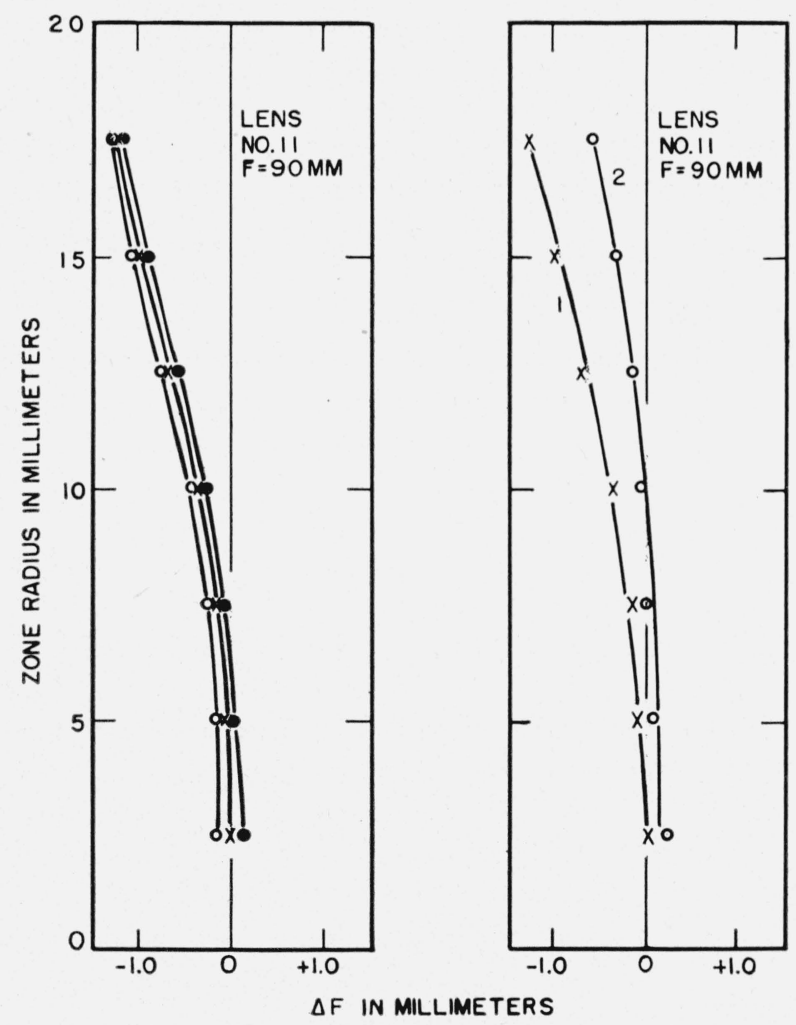

FIGURE 9. Axial longitudinal spherical and axial chromatic aberration curves for a short-focus collimating lens is shown in the left-hand figure.

The open circles are determinations made with Wratten filter No. 75 , the crosses with Wratten filter No. 73, and the solid circles with Wratten filter No. 71A. The right-hand figure shows the effect of astigmatism, curve No. 1 shows the longitudinal spherical aberration along a selected diameter determined with Wratten No. 73 filter, and curve No. 2 shows the results obtained along a diameter at right angles to that used in determining curve No. 1.

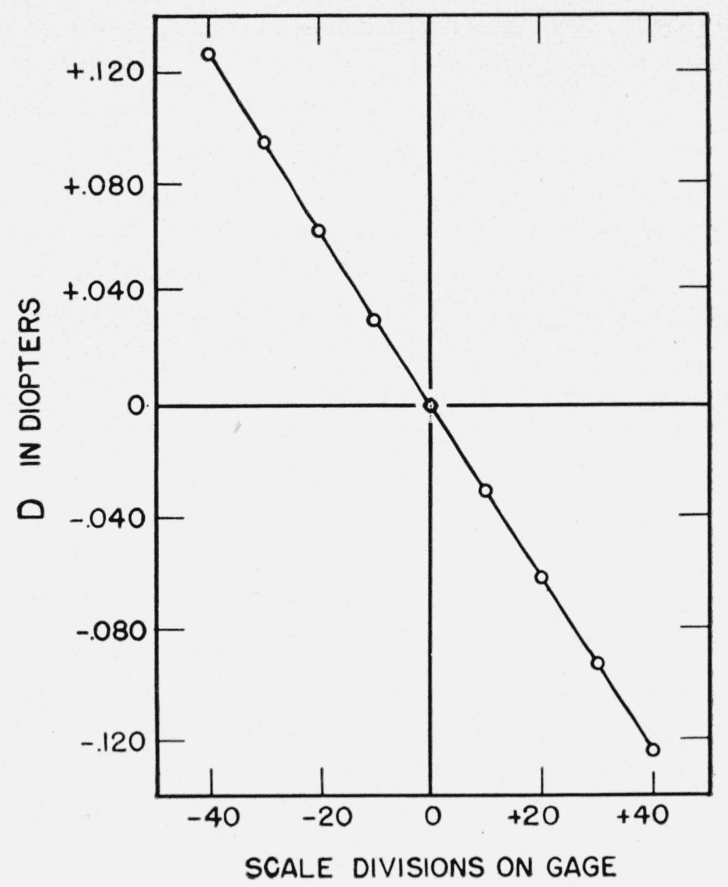

FIgure 10. Calibration curve for the instrument when being used to measure small refractive powers.

on spherical power, which range from 0.00 to +0.06 diopter, are too small to permit accurate determination of compliance on the usual type of lens-testing instrument. The small range of 0.06 diopter amounts to only one-half scale division on most commercial-type instruments.

This new instrument may be readily used for the rapid measurement of small refractive powers. For this condition of use, lens $L_{2}$ is considered a part of the instrument, and its function is the providing of parallel light in the space between $L_{1}$ and $L_{2}$. The instrument is focused for a given pair of diaphragm openings by moving reticle $R$ so that a single image is formed by lens $L_{2}$. The light entering the openings, $O_{1}$ and $O_{2}$, is accordingly parallel. The dial gage is set to read zero for this condition. If a goggle lens is placed over diaphragm openings $O_{1}$ and $O_{2}$ in the parallel light beams, no effect on the image formed by $L_{1}$ is noticed if the goggle lens has zero refracting power. However, if the lens does possess appreciable power, the image splits and reticle $R$ must be moved to cause the two images to coalesce. The change in reading on the gage can then be interpreted in terms of the dioptric power of the goggle lens under test. For example, if the collimating lens, $L_{2}$, has a focal length of $90 \mathrm{~mm}$, and 
the goggle lens has a spherical refracting power of +0.06 diopter, the reticle must move $-0.483 \mathrm{~mm}$ to neutralize the spherical power of the goggle lens. As the dial gage is calibrated in thousandths of an inch, this movement of $0.483 \mathrm{~mm}$ is equivalent to 19 scale divisions on the gage. Figure 10 shows the relation between gage readings and dioptric power of the goggle lens when collimating lens $L_{2}$ has a focal length, $f$, of $90 \mathrm{~mm}$. The points on the curve are located from the relation

$$
D=\frac{-1,000 \Delta f}{f^{2}+f \cdot \Delta f}
$$

where $\Delta f$ is the change in position of reticle $R$, and $D$ is the dioptric power of the goggle lens. With this arrangement, it is possible to measure the dioptric power of the goggle lens to the nearest 0.003 diopter. To determine compliance with the usual tolerances of 0 to +0.06 diopter, it is only necessary to see that the pointer of the gage always lies in the range 0 to -19 scale divisions.

\section{Measurement of Parallax of Collimating Lenses}

When the instrument is used to measure the parallax arising from longitudinal spherical aberrątion in short, focal-length, collimating lenses, such as are commonly used in reflector sights, the problem is mainly the rapid measurement of the maximum and minimum parallax of a lens for compliance with set tolerances, a typical tolerance being \pm 1 mil. Parallax, as here considered, is simply angular spherical aberration resulting from longitudinal spherical aberration, $\Delta f$, in millimeters and is related to it by the formula,

$$
\epsilon=\frac{1019 h \Delta f}{f^{2}} \text { mils, }
$$

where $\epsilon$ is the parallax in mils, $h$ is the radius of the selected zone, in millimeters and $f$ the equivalent focal length of the lens in millimeters.

By proper selection of an intermediate zone and setting the dial gage to read zero when a single image is formed by $L_{1}$ for this zone, the value of $\Delta f$ in the above equation becomes zero for this zone. Positive and negative values of $\epsilon$ can then

Washington, March 31, 1949. be obtained for the other zones with respect to this selected zone. By establishing the condition of zero parallax for the proper intermediate zone, it is possible to obtain maximum positive and maximum negative values of $\epsilon$ approximately equal in magnitude for two other values of the zone radius. The three values of zone radius that satisfy the above conditions will vary from one type of lens to another. However, for a given type of lens the three values of zone radius remain remarkably constant, providing the focal lengths of the various lenses of the given type are approximately equal. The three values of zone radius for a given type of lens are determined initially from a study of the longitudinal spherical-aberration curves of a representative group of lenses of the given type. The curves vary so little from lens to lens that no difficulty is experienced in arriving at an appropriate set of values of zone radius that will be valid for setting the zones of zero, maximum-positive and maximum-negative parallax.

For one type of collimating lens of focal length of $90 \mathrm{~mm}$ and effective aperture of $40 \mathrm{~mm}$, the zone of zero parallax has a radius of $17 \mathrm{~mm}$, the zone of maximum-positive parallax has a radius of $10 \mathrm{~mm}$, and the zone of maximum-negative parallax has a radius of $20 \mathrm{~mm}$. If $\epsilon$ has a value of $\pm 1 \mathrm{mil}$, then $\Delta f$ is equal to $+0.81 \mathrm{~mm}$ for $h=10 \mathrm{~mm}$ and -0.40 for $h=20 \mathrm{~mm}$. Accordingly if a lens of this type is placed on the parallax comparator and focused to yield zero parallax for $h=17$, the zero of the gage may be set under the movable pointer and the two tolerance hands set at +0.81 and $-0.40 \mathrm{~mm}$, respectively. Then, the zones of maximum-positive and negative parallax may be quickly brought into position and the instrument focused for each in turn. If the movable hand does not take a position for either of these settings outside the runge on the dial of the gage marked by the tolerance hands, the parallax does not exceed $\pm 1 \mathrm{mil}$, and the lens is within tolerance. Lenses of this type can be checked for compliance with parallax tolerances at the rate of 30 per hour without placing any strain on the operator. 